\title{
On the relationship between the dual specificity of the bovine brain phosphatidylinositol transfer protein and membrane phosphatidylinositol levels
}

\author{
P.A. Van Paridon ${ }^{\text {a }}$, T.W.J. Gadella, Jr. ${ }^{\text {a }}$, P.J. Somerharju ${ }^{\text {b }}$ \\ and K.W.A. Wirtz ${ }^{\text {a }}$ \\ ${ }^{\triangleleft}$ Laboratory of Biochemistry, State University of Utrecht, Utrecht (The Netherlands) \\ and ${ }^{b}$ Department of Basic Chemistry, University of Helsinki, Helsinki (Finland)
}

(Received 18 March 1987)

Key words: Phosphatidylinositol; Transfer protein; Phospholipid; Membrane phospholipid; (Bovine brain)

The phosphatidylinositol transfer protein from bovine brain has a remarkable specificity pattern with a distinct preference for phosphatidylinositol (PI) and a low affinity for phosphatidylcholine (PC). In this study we have determined the affinity of PI-transfer protein for PI relative to that for PC by measuring the 'sinding of the fluorescent pyrene-labeled analogs of these phospholipids. From competition binding experiments it was estimated that the transfer protein has a 16-fold higher affinity for PI than for PC. This relative affinity together with the relative abundance of $\mathrm{PI}$ and $\mathrm{PC}$, determines what proportion of the protein contains PI (e.g. 65\% of the PI-transfer protein in the case of bovine brain). From measuring lipid transfer between donor vesicles consisting of equimolar amounts of PC and PI, and an excess of acceptor vesicles consisting of various ratios of $P C$ and PI, we have observed that the relative rates of the PI-transfer protein-mediated transfer of PI and PC varies between 5 and 20. Kinetic analysis has indicated that PI-transfer protein carrying a PI molecule has different kinetic properties than the PI-transfer protein carrying a PC molecule. It will be discussed that because of the dual specificity, PI-transfer protein is ideally suited for maintaining PI levels in intracellular membranes.

\section{Introduction}

Phospholipids in cellular membranes are subject to continuous metabolism. The major site of intracellular phospholipid synthesis resides in the endoplasmic reticulum [1-3]. In order to maintain

\footnotetext{
Abbreviations: PI, phosphatidylinositol; PC, phosphatidylcholine; PA, phosphatidic acid; C(16),Pyr(8)-PC, 1-palmitoyl,2pyrenyloctanoyl-sn-glycero-3-phosphocholine; C(16), Pyr(10)PC, 1-palmitoyl,2-pyrenyldecanoyl-sn-glycero-3-phosphocholine; $\mathrm{C}(16)$, Pyr(8)-PI, 1-palmitoyl,2-pyrenyloctanoyl-snglycero-3-phosphoinositol; C(16),Pyr(10)-PI, 1-palmitoyl,2pyrenyldecanoyl-sn-glycero-3-phosphoinositol; TNP-PE, trinitrophenylphosphatidylethanolamine; DMSO, dimethyl-
}

the phospholipid composition of the various cellular membranes, an efficient lipid transfer mechanism must operate. It has been proposed that lipid movement from the endoplasmic reticulum to other intracellular membranes involves vesicular transport via the Golgi system [4]. Phospholipid transfer proteins are also believed to take part in

sulfoxide; Tris, tris(hydroxymethyl)aminomethane; EDTA, ethylenediaminetetraacetic acid; $K_{\text {rel }}$, relative affinity constant.

Correspondence: P.A. van Paridon, Laboratory of Biochemistry, State University of Utrecht, Transitorium III, Padualaan 8, NL-3584 CH Utrecht, The Netherlands. 
the intracellular lipid transport [5-8]. Several studies have indicated that the characteristics of intracellular phospholipid transport are not compatible with a vesicular transport system. In chinese hamster ovary cells for instance, PC transport from the endoplasmic reticulum to the plasma membrane was shown to be extremely fast (halftime of $2 \mathrm{~min}$ at $25^{\circ} \mathrm{C}$ ), hardly temperature dependent, and not affected by energy poisons [7]. Cholesterol transport on the other hand, which is thought to occur by a vesicular mechanism, was found to be relatively slow (halftime of $26.5 \mathrm{~min}$ at $25^{\circ} \mathrm{C}$ ), very temperature dependent and blocked by energy poisons [9]. A study on lipid transport in epithelial cells has indicated that the very rapid transport of PC and PI is in agreement with spontaneous, possibly protein-mediated, phospholipid transport rather than a membrane flow mechanism [10]. It remains to be established whether phospholipid transfer proteins take part in this spontaneous transport of phospholipids.

Recently, a great deal of attention has been focused on the stimulus-induced phopshoinositide metabolism [11,12]. In this respect it is of interest that soluble cytosolic transfer proteins capable of efficient PI transport, have been isolated from a number of tissues [13-17]. This PI-transfer protein exhibits a marked preference for PI, but also transfer PC. PI-transfer protein has been shown to have a low affinity for PG and sphingomyelin $[14,18,19]$, but no affinity for phosphatidic acid, phosphatidylethanolamine or phosphatidylserine $[14,20]$. The transfer protein acts as a soluble lipid-carrier, always containing one endogenous phospholipid molecule $[18,21,22]$. Because of its dual specificity, PI-transfer protein is able to deliver PI to a membrane with a low PI-content, in return for PC, resulting in a net transfer of PI [23]. We assume that this pattern of specificity indicates the presence of individual binding sites for the phosphorylinositol- and phosphorylcholine headgroups.

Intrigued by the unusual substrate specificity pattern of the PI-transfer protein, we have further explored the effect of membrane PI and PC levels on the binding and transfer activity of the PItransfer protein. The data suggest that because of its dual specificity, the PI-transfer protein is very well suited to maintain membrane PI levels.

\section{Materials and Methods}

\section{Materials}

Egg yolk PC and phosphatidic acid (PA) were purchased from Sigma. PI was isolated from bakers' yeast as described by Trevelyan [24], and purified as before [25]. The pyrene-labeled lipid analogs $\mathrm{C}(16) \mathrm{Pyr}(8)-\mathrm{PC}, \mathrm{C}(16) \mathrm{Pyr}(10)-\mathrm{PC}$, $\mathrm{C}(16) \mathrm{Pyr}(8)-\mathrm{PI}$ and $\mathrm{C}(16) \mathrm{Pyr}(10)$-PI were prepared and purified according to Somerharju et al. [26]. Briefly, the lipid analogs were synthesized by acylating the lyso-compounds, prepared from di$\mathrm{C}(16)-\mathrm{PC}$ and yeast PI, using anhydrides of the pyrene-fatty acids. In the case of PI, the hydroxyl groups were protected by acetylation. The molecular species of PI containing a palmitoyl acyl chain in the $s n-1$ position were isolated by reversed phase HPLC. Trinitrophenyl-phosphatidylethanolamine (TP-PE) was prepared from egg yolk PE and trinitrobenzene sulfonic acid [27]. PI-transfer protein was isolated from bovine brain [22], and stored in $50 \%$ glycerol at $-20^{\circ} \mathrm{C}$.

\section{Methods}

The concentration of the PyrPC and PyrPI species was determined by measuring the fluorescence intensity in DMSO (Merck, Uvasol) (excitation at $346 \mathrm{~nm}$, emission at $378 \mathrm{~nm}$, slits 5 and 1 $\mathrm{nm}$, respectively). The fluorescence intensity was callibrated with a C(16)Pyr(10)-PC standard of which the concentration had been established by phosphorus determination [28].

Preparation of phospholipid vesicles. Donor vesicles were prepared by injection of an ethanolic lipid solution (15 $\mu 1,0.1-10 \mathrm{nmol}$ total lipid) into $2 \mathrm{ml}$ of $20 \mathrm{mM}$ Tris- $\mathrm{HCl}, 5 \mathrm{mM}$ EDTA, $200 \mathrm{mM}$ $\mathrm{NaCl}$ ( $\mathrm{pH}$ 7.4) [29]. The donor lipids consisting of mixtures of pyrene-labeled and unlabeled phospholipids and TNP-PE were dissolved in DMSO (less than 25\% of total volume) before the ethanol was added. Prior to use, the donor vesicles were allowed to equillibrate for $2 \mathrm{~min}$. Acceptor vesicles consisting of various amounts of PI and PC were prepared by ultrasonication of a lipid emulsion ( 2 $\mathrm{mM}$ lipid) in the Tris-EDTA- $\mathrm{NaCl}$ buffer under a nitrogen atmosphere, with a Branson sonifier (5 $\left.\min , 0^{\circ} \mathrm{C}, 50 \mathrm{Watt}\right)$. The buffer was routinely filtered through a millipore filter $(0.45 \mu \mathrm{m})$.

Fluorescence measurements. Lipid-binding and 
transer assays were carried out using a SLMAminco SPF-500C spectrofluorometer equipped with a thermostated cuvette holder and a magnetic stirring device. Excitation was set at $346 \mathrm{~nm}$ (slit 2 $\mathrm{nm}$ ), and the pyrenyl-monomer fluorescence was monitored at $378 \mathrm{~nm}$ (slit $10 \mathrm{~nm}$ ). All measurements were performed at $37^{\circ} \mathrm{C}$ with continuous stirring.

Binding assay. Binding of PyrPC and PyrPI was measured by titration of donor vesicles $(0.1-10$ nmol phospholipid) with PI-transfer protein (1-10 $\mu \mathrm{g})$ in $2 \mathrm{ml}$ of buffer. The vesicles consisted of PyrPC or PyrPI diluted with varying amounts of unlabeled PC or PI. In all cases TNP-PE was added (10 mol\%) to effectively quench the pyrenyl fluorescence signal in the donor vesicles. The increase in pyrene monomer fluorescence intensity resulting from the uptake of PyrPC or PyrPI from the donor vesicles by PI-transfer protein, was taken as a measure for pyrenyl lipid binding (Somerharju et al., manuscript submitted).

Transfer assay. Lipid transfer was determined by following the pyrene monomer fluorescence increase upon the addition of PI-transfer protein $(1 \mu \mathrm{g})$ to a mixture of quenched donor vesicles and an excess (20-100-fold) of unlabeled acceptor vesicles in $2 \mathrm{ml}$ of buffer (Ref. 30 and Van Paridon et al., manuscript submitted). A correction was made for passive transfer. In order to be able to directly compare efficiency for PyrPI and PyrPC, the donor vesicles consisted of equimolar amounts of either PyrPI and unlabeled PC, or PyrPC and unlabeled PI.

Determination of relative affinity constants. In order to determine the affinity constants for PyrPC and PyrPI relative to egg $\mathrm{PC}$ and yeast PI, pyrenyl lipid binding was measured using donor vesicles consisting of a fixed amount of labeled lipid and variable amounts of unlabeled lipid. By this experimental approach, competition is measured between labeled and unlabeled lipid for binding to PI-transfer protein.

When PI-transfer protein binds a labeled lipid molecule $\left(\mathrm{L}_{1}\right)$, or an unlabeled lipid molecule $\left(\mathrm{L}_{0}\right)$, it is designated as $\mathrm{P}_{1}$ or $\mathrm{P}_{0}$, respectively. Since, in all experiments, the total PI-transfer protein concentration is very low relative to the donor lipid concentration, we may assume that binding of $\mathrm{L}_{0}$ and $L_{1}$ to PI-transfer protein nor the release of the endogenous lipids has any effect on the lipid composition of the donor membrane. Binding of the species $\mathrm{L}_{0}$ and $\mathrm{L}_{1}$ can be presented by the following equilibrium

$\mathrm{L}_{1}+\mathrm{P}_{0} \stackrel{K_{\text {red }}}{\rightleftarrows} \mathrm{P}_{1}+\mathrm{L}_{0}$

The equilibrium constant $K_{\text {rel }}$ represents the binding affinity of PI-transfer protein for $\mathrm{L}_{1}$ relative to $\mathrm{L}_{0}$ (Eqn. 2)

$K_{\text {rel }}=\frac{\left[\mathrm{P}_{1}\right] \cdot\left[\mathrm{L}_{0}\right]}{\left[\mathrm{P}_{0}\right] \cdot\left[\mathrm{L}_{1}\right]}=\frac{\left[\mathrm{P}_{1}\right] \cdot\left[\mathrm{L}_{0}\right]}{\left(\left[\mathrm{P}_{\text {tot }}\right]-\left[\mathrm{P}_{1}\right]\right) \cdot\left[\mathrm{L}_{1}\right]}$

By rearranging Eqn. 2 we obtain

$\left[\mathrm{P}_{1}\right]=\frac{-1}{K_{\mathrm{rel}}} \cdot \frac{\left[\mathrm{L}_{0}\right]}{\left[\mathrm{L}_{1}\right]} \cdot\left[\mathrm{P}_{1}\right]+\left[\mathrm{P}_{\mathrm{tot}}\right]$

In the binding assay the increase in pyrene monomer fluorescence $(\Delta F)$ is proportional to the amount of $\mathrm{L}_{1}$ bound to PI-transfer protein. In the absence of unlabeled lipid in the donor vesicles $\left(\left[\mathrm{L}_{0}\right]=0\right)$, a maximal fluorescence increase $\left(\Delta F_{\max }\right)$ is observed $\left(\left[\mathrm{P}_{\text {tot }}\right]=\left[\mathrm{P}_{1}\right]\right)$. Therefore Eqn. 3 can be presented as follows

$\Delta F=\frac{-1}{K_{\mathrm{rel}}} \cdot \frac{\left[\mathrm{L}_{0}\right]}{\left[\mathrm{L}_{1}\right]} \cdot \Delta F+\Delta F_{\max }$

By plotting $\Delta F$ as a function of $\Delta F \cdot\left[\mathrm{L}_{0}\right] /\left[\mathrm{L}_{1}\right]$, a straight line is obtained with a slope of $-1 / K_{\text {rel }}$.

The $K_{\text {rel }}$ value was calculated by a least-squares optimization of the experimental data.

\section{Results}

\section{Binding of PyrPC and PyrPI}

Binding of PyrPC and PyrPI was determined by titration of quenched donor vesicles with PItransfer protein. A typical titration experiment is shown in Fig. 1. The monomer and excimer fluorescence in the donor vesicles were strongly quenched due to the presence of TNP-PE. Upon addition of PI-transfer protein an increase in pyrene-monomer fluorescence $\left(\Delta F, \lambda_{\max } 378 \mathrm{~nm}\right)$ is observed proportional to the amount of pyrenyl lipid bound.

In order to determine the affinity of PI-transfer protein for $\mathrm{C}(16)$ Pyr(10)-PI relative to that for egg 


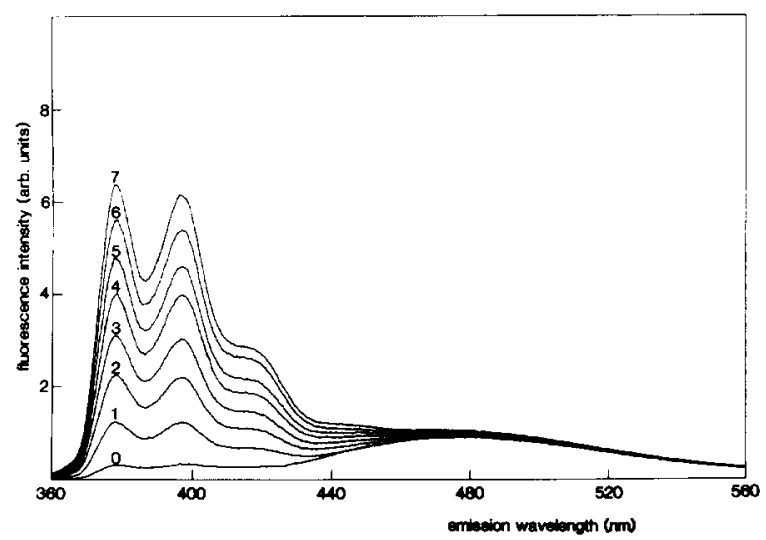

Fig. 1. Fluorescent lipid binding assay. Lipid binding was quantitated by measuring the increase in pyrene-monomer fluorescence (maximum at $378 \mathrm{~nm}$ ), using $1.0 \mathrm{nmol}$ of quenched donor vesicles (C(16),Pyr(10)-PC/TNP-PE; $90: 10 \mathrm{~mol} \%$ ) titrated with PI-transfer protein ( $1 \mu \mathrm{g}$ aliquots).

PC, a series of donor vesicles was prepared consisting of a constant amount $(0.162 \mathrm{nmol})$ of $\mathrm{C}(16) \mathrm{Pyr}(10)-\mathrm{PI}$, and increasing amount egg PC $(0-6 \mathrm{nmol})$. From the titration curves (see Fig. 2A) it is apparent that egg PC cannot effectively compete with PyrPI. For instance, dilution of C(16)Pyr(10)-PI with a ten-fold excess of egg PC diminished binding by only a factor of two. In Fig. $2 \mathrm{~B} \Delta F$ is plotted as a function of $\left(\left[\mathrm{L}_{0}\right] /\left[\mathrm{L}_{1}\right]\right)$.
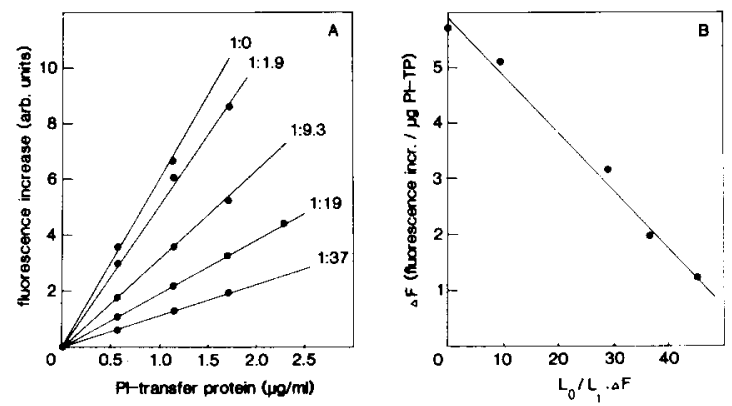

Fig. 2. Determination of the affinity constant $\left(K_{\text {rel }}\right)$ of PItransfer protein for $\mathrm{C}(16), \operatorname{Pyr}(10)-\mathrm{PI}$ relative to egg $\mathrm{PC}$. Quenched donor vesicles consisted of a fixed amount of C(16), Pyr(10)-PI (0.162 nmol), variable amounts of egg PC, and TNP-PE (10 mol\%). Lipid binding is presented as the increase in monomer fluorescence as a function of the PI-transfer protein concentration (A). The numbers in the graph correspond to the ratio of PyrPI to egg PC in the vesicles. B. The slopes of the lines in (A) $(\Delta F / \mu \mathrm{g}$ PI-transfer protein) are plotted as a function of the ratio of unlabeled to labeled lipid multiplied by the slope, as descriebd by Eqn. 4 in Materials and Methods.
$\Delta F$. As predicted by the model described in Materials and Methods a linear relationship was observed. From the plot a $K_{\text {rel }}$ value of $9.69 \pm 0.51$ was obtained. This value demonstrates that PItransfer protein has a ten-fold higher affinity for C(16)Pyr(10)-PI than for egg PC.

To further investigate the affinity for PI compared to PC, several sets of donor vesicles were prepared. The vesicles consisted of either C(16)Pyr(8)-PC, C(16)Pyr(10)-PC, C(16)Pyr(8)-PI or $\mathrm{C}(16) \mathrm{Pyr}(10)$-PI diluted with varying amounts of unlabeled PC or PI. The binding of $\mathrm{C}(16) \mathrm{Pyr}(10)-\mathrm{PC}$ and $\mathrm{C}(16) \mathrm{Pyr}(10)$-PI as affected by the presence of unlabeled phospholipids are shown in Fig. 3. Here, the binding of pyrenyl-lipid is presented as a function of the the unlabeled lipid/pyrenyl-lipid ratio $\left(\mathrm{L}_{0} / \mathrm{L}_{1}\right)$. Dilution of PyrPI with egg PC (top curve), resulted in a gradual decrease in binding of the fluorescent lipid (see also Fig. 2). When this lipid was diluted with yeast PI, a more pronounced decrease in binding was observed. A 50\% dilution of the PyrPI with yeast PI resulted in an approximately 2 -fold reduction in PyrPI binding, indicating a rather similar binding affinity for yeast PI and C(16)Pyr(10)-PI. A similar binding affinity was also observed for C(16)Pyr(10)-PC and egg PC. A

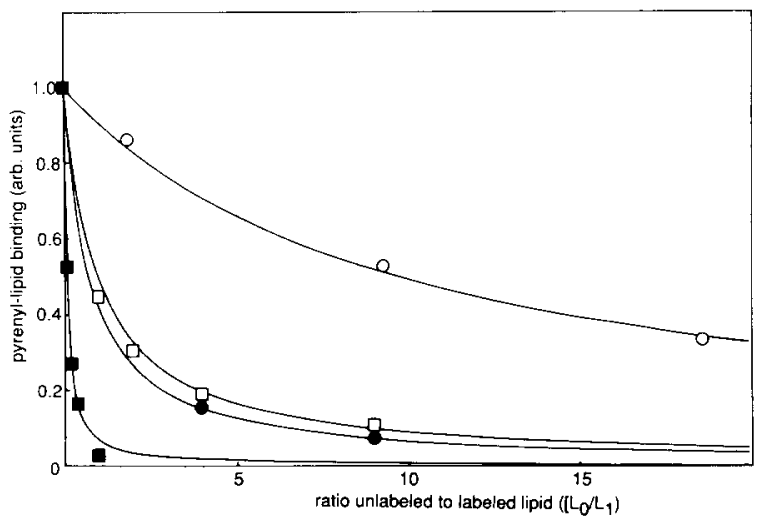

Fig. 3. The effect of unlabeled lipids in the donor vesicles on the binding of pyrene-labeled lipids by PI-transfer protein. Pyrenyl-lipid binding was measured as described in the legend of Fig. 1, using quenched donor vesiles consisting of Pyr(10)-PI $(\bullet, 0)$ or Pyr $(10)$-PC $(\square, \square)$ and varying amounts of unlabeled egg PC $(O, \square)$ or yeast PI $(\bullet, \square)$. Binding of the pyrenyl-lipids is represented normalized to the value obtained with pure pyrenyl-lipid, as a function of the ratio of unlabeled $\left(\mathrm{L}_{0}\right)$ to labeled lipid $\left(\mathrm{L}_{1}\right)$. 
very dramatic decrease in binding was observed when $\mathrm{C}(16) \mathrm{Pyr}(10)$-PC was diluted with yeast PI (Fig. 3, bottom curve). This decrease is caused by the much higher affinity of PI-transfer protein for yeast PI than for $\mathrm{C}(16) \mathrm{Pyr}(10)$-PC. In agreement with the specificity of PI-transfer protein dilution of C(16)Pyr(10)-PC with an equimolar amount of PA did not result in a decrease in binding. This implies that an increase of the vesicle surface charge per se has no effect on the binding properties of PI-transfer protein. The differences observed in the binding of PI and PC cannot be attributed to a different accessibility of these lipids since PI and PC distribute equally over the inner and outer leaflet of the membrane of small unilamellar vesicles [31]. Results similar to those in Fig. 3 were obtained with $\mathrm{C}(16) \operatorname{Pyr}(8)-\mathrm{PC}$ and C(16)Pyr(8)-PI (data not shown). From the binding data, the $K_{\text {rel }}$ values for the pyrenyl lipids relative to the unlabeled lipids were determined, as described in Materials and Methods (see Table I). It is evident that PI-transfer protein has a similar affinity for PyrPC and egg PC ( $K_{\text {rel }}$ of $1.0-1.2)$, as well as for PyrPI and yeast PI ( $K_{\text {rel }}$ of $0.7-0.8)$, indicating that the presence of a $\operatorname{Pyr}(8)$ or Pyr(10)-acyl-chain does not significantly influence the binding affinity of PI-transfer protein. On the other hand, PI-transfer protein clearly prefers PyrPI over egg PC, and yeast PI over PyrPC.

Up to now we have described the affinity of PI-transfer protein for pyrenyl lipids relative to

\section{TABLE I}

RELATIVE AFFINITY CONSTANTS OF PI-TRANSFER PROTEIN FOR Pyr(8)- AND Pyr(10)-PI AND -PC COMPARED TO YEAST PI AND EGG PC

The relative affinity constants $\left(K_{\text {rel }}\right)$ were determined in competition binding experiments as described in Materials and Methods.

\begin{tabular}{lcl}
\hline $\begin{array}{l}\text { Labeled phospho- } \\
\text { lipid }\left(\mathrm{L}_{1}\right)\end{array}$ & \multicolumn{2}{c}{$K_{\text {rel }}$, Nonlabeled phospholipid $\left(\mathrm{L}_{0}\right)$} \\
\cline { 2 - 3 } & \multicolumn{1}{l}{ egg PC } & yeast PI \\
\hline 1-Pal,2-Pyr(8)-PC & $1.17 \pm 0.23$ & $0.0608 \pm 0.0078$ \\
1-Pal,2-Pyr(10)-PC & $0.98 \pm 0.13$ & $0.074 \pm 0.015$ \\
1-Pal,2-Pyr(8)-PI & $13.77 \pm 0.97$ & $0.790 \pm 0.011$ \\
1-Pal,2-Pyr(10)-PI & $9.69 \pm 0.51$ & $0.713 \pm 0.03$ \\
\hline
\end{tabular}

unlabeled lipids. However, from the $K_{\text {rel }}$ values in Table I the affinity of PI-transfer protein for yeast PI relative to egg PC can be calculated (see Fig. 4). For example

$K_{\text {rel }}\left(\frac{\text { yeast PI }}{\text { egg PC }}\right)=K_{\text {rel }}\left(\frac{\text { yeast PI }}{\text { PyrPI }}\right) \cdot K_{\text {rel }}\left(\frac{\text { PyrPI }}{\text { egg PC }}\right)\left(\right.$ i.e. $\left.a^{-1} \cdot b\right)$

This affinity value can also be determined via PyrPC $\left(c \cdot d^{-1}\right)$. Since four pyrenyl lipids were used in this studyl, four independent $K_{\text {rel }}$ values for yeast PI relative to egg PC could be estimated, yielding an average $K_{\text {rel }}$ value of $15.6 \pm 3.8$ (S.D.).

\section{Transfer of PyrPC and PyrPI}

Ratios for PI transfer over PC transfer were reported to vary between 4 and $20(14,17,19)$. These observations raise questions on how the sixteen-fold higher affinity of PI-transfer protein for $\mathrm{PI}$ relative to $\mathrm{PC}$ relates to the relative transfer rates for these two phospholipids. Here, we have investigated this problem by measuring pyrenylphospholipid transfer from donor vesicles consisting of equimolar amounts of PI and PC (1.1 nmole phospholipid), to an excess of acceptor vesicles (20-100 fold). Under these experimental conditions, the relative rates of PyrPI and PyrPC transfer are determined by the exchange process at the donor membrane. The donor vesicles consisted of PyrPC, yeast PI and TNP-PE $(45: 45: 10$ mole\%), or PyrPI, egg PC and TNP-PE (45: $45: 10$ mole\%). By using acceptor vesicles consisting of PC-PA (95:5 mole\%) or PI (100 mole\%), relative transfer rates for PyrPI and PyrPC were found to

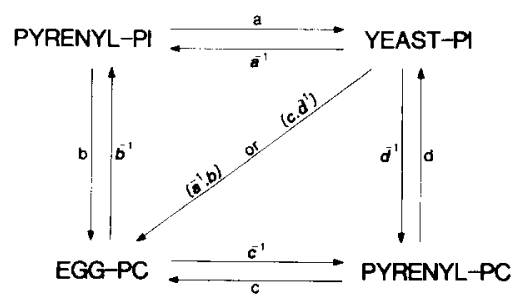

Fig. 4. Protocol for the calculation of the affinity constant of PI-transfer protein for yeast PI relative to egg PC. The $K_{\text {rel }}$ values for the pyrenyl-lipid compared to the unabeled lipid are represented by $a, b, c$ and $d$. The affinity for an unlabeled lipid relative to a labeled lipid is represented by the reciprocal values (i.e. $a^{-1}, b^{-1}, c^{-1}$ and $d^{-1}$ ). The affinity for yeast PI relative to that for egg $\mathrm{PC}$ is given by $\left(a^{-1} \cdot b\right)$ or $\left(c \cdot d^{-1}\right)$. 
be 5 and 20, respectively (see Fig. 5). This difference must be due to the fact that upon arrival at the donor membrane PI-transfer protein contains PC in the case of the PC-acceptor vesicles, or PI in the case of the PI-acceptor vesicles. Apparently, the phospholipid bound to PI-transfer protein affects the relative rates at which this endogenous lipid is exchanged for PyrPI or PyrPC. This interpretation was confirmed by measuring transfer to acceptor vesicles consisting of $\mathrm{PC}$ and 10 or $50 \mathrm{~mol} \%$ of PI, yielding relative transfer rates for PyrPI over PyrPC with values in between 5 and 20 (see Fig. 5). Under these conditions the proportion of PI-transfer protein containing an endogenous PI or PC molecule varies depending on the lipid composition of the acceptor membranes. It is to be noted that decreasing the pyrenyl acyl chain length by two methylene units results in an increase of PyrPI transfer relative to PyrPC transfer. As a control, transfer was also measured to vesicles consisting of PC-PA (50:50 mol\%).

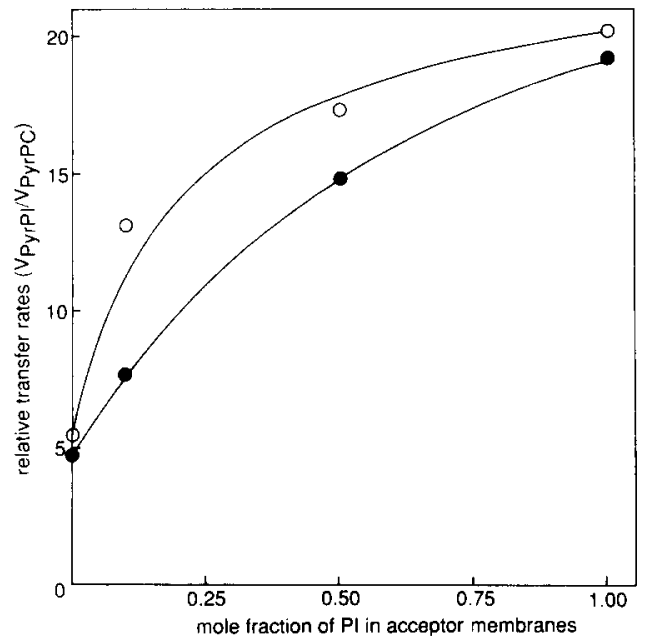

Fig. 5. The transfer specificity of PI-transfer protein as a function of the molar ratio of $\mathrm{PI}$ to $\mathrm{PC}$ in the acceptor membrane. Transfer of PyrPI and PyrPC were measured between quenched donor vesicles containing equal amounts of either PyrPI and egg PC, or of yeast PI and PyrPC (1.1 nmol of total phospholipid), and acceptor vesicles (20-100-fold excess) consisting of yeast PI and egg PC in various ratios. The transfer rates of $\mathrm{Pyr}(8)$-PI relative to those of $\mathrm{Pyr}(8)-\mathrm{PC}(\mathrm{O})$, and of Pyr(10)PI relative to those of $\operatorname{Pyr}(10)-\mathrm{PC}(\bullet)$ are shown. The curves are calculated by substitution of the relative affinity constants (see Table I) and the proportion of PI-TP containing an endogenous PI or PC molecule (Eqn. A-10 from the appendix) into Eqn. A-6 (see appendix).
Relative transfer rates were identical to those found for PC vesicles containing $5 \mathrm{~mol} \% \mathrm{PA}$, in support of our conclusion that thePI/PC ratio in the acceptor vesicles determines the transfer specificity.

In our experiments transfer of PyrPI and PyrPC was measured using donor vesicles with identical physical properties, e.g. identical in PC content, PI content, surface charge and pyrenyl-acyl chain content. In the presence of a large excess of acceptor vesicles, the rate-limiting step in the transfer process between donor and acceptor membranes is the exchange of a protein-bound lipid molecule for a pyrenyl lipid molecule from the donor membrane. Therefore, differences in PyrPI and PyrPC transfer rates are a reflection of the exchange rates of these lipids at the donor membrane. The equations that describe the exchange reactions at the donor membrane are presented in the appendix. From the relative rates of PyrPI and PyrPC transfer, and the proportion of PI-transfer protein containing a PI or a PC molecule, the relative rate constants for the four possible exchange reactions at the donor vesicle can be estimated (see Table

\section{TABLE II}

\section{RELATIVE RATE CONSTANTS FOR THE EXCHANGE OF PI-TRANSFER PROTEIN-BOUND PHOSPHOLIPID FOR DONOR VESICLES PHOSPHOLIPID}

Relative rate constants for the four possible exchange reactions at the donor membrane measured with either Pyr(8)-lipids or Pyr(10)-lipids, and calculated using the data from Fig. 5 as described in the appendix. PI-transfer protein containing an endogenous lipid molecule is represented as $P_{(x)}$, where $x$ stands for the endogenous lipid molecule. The donor vesicles are represented as D. $k_{1}-k_{4}$ are the relative rate constants for the four exchange routes.

\begin{tabular}{lll}
\hline $\begin{array}{l}\text { Exchange reactions } \\
\text { at the donor membrane }\end{array}$ & \multicolumn{2}{l}{ Rate constants (relative to $k_{4}$ ) } \\
\cline { 2 - 3 } $\begin{array}{l}\mathrm{Pyr}(8)- \\
\text { lipids }\end{array}$ & $\begin{array}{l}\text { Pyr(10)- } \\
\text { lipids }\end{array}$ \\
\hline $\mathrm{P}_{(\mathrm{PC})}+\mathrm{D} \stackrel{k_{1}}{\rightarrow} \mathrm{P}_{(\mathrm{PyrPC})}+\mathrm{D}$ & 0.13 & 0.35 \\
$\mathrm{P}_{(\mathrm{PC})}+\mathrm{D} \stackrel{k_{2}}{\rightarrow} \mathrm{P}_{(\mathrm{Pyr} \mathrm{PI})}+\mathrm{D}$ & 0.72 & 1.7 \\
$\mathrm{P}_{(\mathrm{PI})}+\mathrm{D} \stackrel{k_{3}}{\rightarrow} \mathrm{P}_{(\mathrm{Pyr} \mathrm{PC})}+\mathrm{D}$ & 0.05 & 0.05 \\
$\mathrm{P}_{(\mathrm{PI})}+\mathrm{D} \stackrel{k_{4}}{\rightarrow} \mathrm{P}_{(\mathrm{Pyr} \mathrm{PI})}+\mathrm{D}$ & 1.00 & 1.00 \\
\hline
\end{tabular}


II). Independent of whether PI-transfer protein contains PI or PC, binding of both PyrPI analogs proceeds at a comparable rate. On the other hand, binding of PyrPC is 20-times slower when PItransfer protein carries an endogenous PI molecule and 3-7-times slower when it carries a PC molecule. It is evident that the exchange of endogenous $\mathrm{PC}$ for $\operatorname{Pyr}(8)$-PC and $\operatorname{Pyr}(8)$-PI is 2-3-times slower than for Pyr(10)-PC and Pyr(10)-PI. Substitution of the rate constants into Eqn. 6 (see appendix) yielded the curves drawn in Fig. 5. There was an excellent fit with the experimental data points for $\operatorname{Pyr}(10)-\mathrm{PC}$ and -PI; the fit was somewhat less for the $\mathrm{Pyr}(8)$ analogs.

These results indicate that the endogenous lipid molecule influences the kinetic properties of PItransfer protein, thereby affecting the relative rates at which PI and PC are transferred.

\section{Discussion}

In this study we have used the pyrene-labeled analogs of PI and PC to determine the binding and transfer of these phospholipids by PI-transfer protein. From the binding experiments we have been able to estimate that the affinity of PI-transfer protein is sixteen-fold higher for PI than for $P C$. This difference in affinity is caused by the interaction of the protein with the polar headgroup, since the accomodation of the acyl chains of both PI and PC on the protein is very similar (Ref. 22 and Van Paridon et al., manuscript submitted). Modification of the inositol ring by periodate oxidation and subsequent reduction by sodium borohydride, or phopshorylation at the 4-position, greatly reduced the transport by PI transfer protein [18,32]. This supports the notion that a specific binding site for the phosphorylinositol headgroup exists.

In a great number of studies the specificity of PI-transfer protein has been investigated by measuring transfer of phospholipids. In all these studies PI was the preferred substrate, but, relative to $\mathrm{PC}$, the transfer rates varied from 1.5 to 20 $[13,14,16,17,19,33]$. In the present study we have established that, under conditions where the donor phospholipid pool is rate-limiting, the relative rates of PI and PC transfer are a function of the acceptor $\mathrm{PI} / \mathrm{PC}$ content. The relative amounts of PI and $\mathrm{PC}$ in the acceptor membrane, in conjunction with the relative affinity of PI-transfer protein for PI and PC ( $K_{\text {rel }}$ of 16$)$, determines the ratio of PI and PC bound to PI-transfer protein. Analysis of the transfer data had clearly shown that PI-transfer protein carrying PI has different kinetic properties than PI-transfer protein carrying PC. This difference must be due to the fact that the rate at which protein-bound PI exhanges for PI and PC from the interface, differs from the rate at which protein-bound PC is exchanged (see Table II). From this analysis we can now understand the various specificity ratios reported by other laboratories. In agreement with our results values between 5 and 20 have been reported repeatedly $[18,33]$. The use of donor membranes with a low $\mathrm{PI} / \mathrm{PC}$ ratio gave rise to even lower transfer ratios $[13,21,23,33]$. In determining the transfer specificity of PI-transfer protein it is important to realize that phospholipid composition and the proportion of acceptor to donor phospholipid has a great effect on the actual transfer ratios measured. In the kinetic model proposed by Helmkamp et al. [21] for the action of PI-transfer protein, different kinetic properties were also ascribed to PI-transfer protein with or without an endogenous PI molecule. In that study, however, the ratio of PI and $\mathrm{PC}$ present in the membranes was not considered to have a direct effect on the kinetic behaviour of PI-transfer protein. On the other hand, our simplified model only gives information on the relationship between PyrPI and PyrPC transfer.

In a previous study from this laboratory using phospholipid monolayers, the the rate at which PI-transfer protein exchanged its bound phospholipid for a molecule from the interface, were determined [20]. It was shown that protein-bound PC exchanged twenty times faster for PI than protein-bound PI for PC. This is in good agreement with the rate constants derived from our kinetic experiments, which gave a factor of 15 for the $\operatorname{Pyr}(8)$-lipids and of 35 for the Pyr(10)-lipids (see Table II). However, we have found that the exchange of protein-bound PC for PI is in the same order as that of protein-bound PI, while the monolayer experiments have indicated that the exchange of protein-bound PI for PI is almost a factor of ten lower. This discrepancy may be due to the fact that in the monolayer experiments 
PI-transfer protein has to approach an interface consisting of pure PI, while in the transfer experiments it has to interact with donor vesiles consist of equimolar amounts of PC and PI.

Upon isolation of PI-transfer protein from bovine brain, approx. $65 \%$ of the protein was found to contain an endogenous PI molecule [22]. From the PI to PC ratio of 0.11 in a bovine brain homogenate (34) and from the $K_{\text {rel }}$ value of 15.6 , one can calculate that $63 \%$ of the PI-transfer protein should contain an endogenous PI molecule. This excellent agreement with the observed value indicates that PI-transfer protein has a marked preference for PI in the cell as well. In view of its phospholipid transfer properties, we presume that PI-transfer protein is involved in the maintainance of intracellular PI-levels. Under conditions where the PI-PC ratio in the donor and the acceptor vesicle is identical, the PI-transfer protein mediated transfer of PI from donor to acceptor equals the transfer of PI in the opposite direction. When the PI-PC ratio is different in the two vesicle populations, a redistribution of $\mathrm{PI}$ occurs as a result of net transfer which is compensated for by a net transfer of PC in the opposite direction [23]. Net transfer of PI by PI-transfer protein in the cell will be determined by the $K_{\text {rel }}$ value of 16 , and by the PI-PC ratios of the various subcellular membranes. If we assume an overall PI-PC ratio in the cell of 0.1 , and a reduction of this ratio in the plasma membrane to 0.05 as a result of stimulus-induced phosphoinositide turnover, we can estimate that the relative PI content of PI-transfer protein is reduced by $17 \%$ upon leaving the plasma membrane, as compared to its PI content upon arrival at this membrane. This implies that $17 \%$ of the lipid transfer to the plasma membrane is net transfer of PI superimposed on a continuous exchange process. In Fig. 6 we show how in the above example the net transfer of PI depends on the $K_{\text {rel }}$ value. It is evident that an optimal net transfer of PI occurs at a $K_{\text {rel }}$ value of 14 . Net transfer of PI is minimal when the $K_{\text {rel }}$ value is either too low $(<1)$, or too high ( $>100$ ). This strongly suggests that with the $K_{\text {rel }}$ value of 15.6 estimated in our study, PI-transfer protein is optimally sensitive towards changes in the plasma membrane PI-PC ratio in the range of 0.1-0.05. As shown in Fig. 6, it appears that the

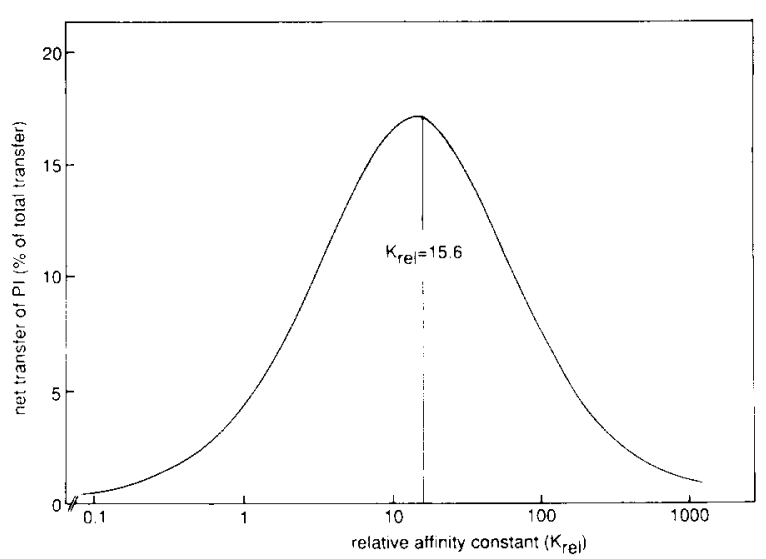

Fig. 6. Dependence of the net transfer of PI to a membrane deficient in PI (e.g. the plasma membrane after stimulus-induced PI breakdown) on the relative affinity constant $\left(K_{\text {rel }}\right)$ of PI-transfer protein. The PI/PC ratio in the donor membrane (e.g. the endoplasmic reticulum), and in the acceptor membrane (e.g. the plasma memrbane deficient in PI) is 0.1 and 0.05 , respectively (see Discussion for the choice of the PI/PC ratios). The net transfer of $\mathrm{PI}$ is equal to the difference in PI content of PI-transfer protein upon arrival and departure at the acceptor membrane. The PI content of PI-transfer protein can be calculated using Eqn. A-10 (appendix). Upon arrival, the $\mathrm{PI}$ content is determined by the $\mathrm{PI} / \mathrm{PC}$ ratio of the donor membrane, and upon departure by the $\mathrm{PI} / \mathrm{PC}$ ratio in the acceptor membrane.

affinity of PI-transfer protein for PI relative to that for $\mathrm{PC}$ is optimal for maintaining the $\mathrm{PI} / \mathrm{PC}$ ratios in the range of 0.05 to 0.1 , prevalent in many biological membranes. For example, the $\mathrm{PI} / \mathrm{PC}$ ratio in bovine brain membranes is 0.11 [34]. This ratio could be substantially decreased in the plasma membane by stimulus induced PI breakdown. A decrease of $50 \%$ has been reported in rat liver plasma membranes [35]. It still remains to be established whether PI-transfer protein is involved in the transfer of PI to the plasma membrane. Studies on yeast cells have clearly indicated that the transport of PI to the plasma membrane is not linked to intracellular vesicle movement, required for protein translocation [8]. In this respect it is of interest that a PI-transfer protein has been detected in yeast [15]. Detailed quantitative analysis of the pathways for phospholipid biosynthesis has convincingly demonstrated that the plasma memrbanes in rat liver lack the capacity for de novo PI synthesis [36]. In a recent study evidence was provided indicating that the plasma membranes of rat pituitary tumour $\left(\mathrm{GH}_{3}\right)$ cells do 
have the ability to synthesize PI from exogenous CDP-diacylglycerol [37]. This suggests that PItransfer protein would not be necessary in this system for the maintenance of PI levels. On the other hand, it was shown in this study that hormonal stimulation of PI synthesis occurred both in the plasma membrane and the endoplasmic reticulum to exactly the same extent. This would be in support of the notion that the PI-pools in the various intracellular membranes are in equilibrium, possibly due to PI-transfer protein. One would expect that rapid restoration of plasma membrane PI-levels is a prerequisite for the proper functioning of excitable tissues. In this respect it is of interest that PI-transfer protein is relatively abundant in brain and heart [33].

Despite the remaining uncertanties, it appears that the specificity of PI-transfer protein is optimal for maintaining the steady-state levels of PI in the intracellular membranes, and to respond to sudden stimulus-induced changes in the PI content of the plasma membrane.

\section{Appendix}

The uptake of a lipid molecule from the donor vesicle can be described by the following equations

$\mathrm{P}_{(\mathrm{PC})}+\mathrm{D} \stackrel{k_{1}}{\rightarrow} \mathrm{P}_{(\mathrm{Pyr} P C)}+\mathrm{D}$

$\mathrm{P}_{(\mathrm{PC})}+\mathrm{D} \stackrel{k_{2}}{\rightarrow} \mathrm{P}_{(\mathrm{Pyr} \mathrm{Pl})}+\mathrm{D}$

$\mathrm{P}_{(\mathrm{PI})}+\mathrm{D}^{k_{3}} \rightarrow \mathrm{P}_{(\mathrm{Pyr} \mathrm{PC})}+\mathrm{D}$

$\mathrm{P}_{(\mathrm{PI})}+\mathrm{D}^{k_{4}} \rightarrow \mathrm{P}_{(\mathrm{Py} \mathrm{PI})}+\mathrm{D}$

in which $\mathrm{P}_{(\mathrm{PI})}$ and $\mathrm{P}_{(\mathrm{PC})}$ represents a PI-transfer protein molecule containing an endogenous PI or PC molecule, and $k_{i}$ are the rate constants for the exchange of a protein-bound lipid molecule for a pyrene labeled lipid from the interface. The ratio of PyrPI to PyrPC transfer from donor to acceptor vesicles is determined by the exchange process at the donor vesicle. This is the rate limiting step in the transfer process, as the donor vesicles concentration in one to two orders of magnitude lower than the acceptor vesicle concentation. Hence, the transfer ratio is given by

$$
\frac{V_{(\mathrm{Pyr} P \mathrm{I})}}{V_{(\mathrm{Pyr} \mathrm{PC})}}=\frac{k_{2} \cdot\left[\mathrm{P}_{(\mathrm{PC})}\right] \cdot\left[\mathrm{D}_{(\mathrm{PyrPI})}\right]+k_{4} \cdot\left[\mathrm{P}_{(\mathrm{PI})}\right] \cdot\left[\mathrm{D}_{(\mathrm{PyrPI})}\right]}{k_{1} \cdot\left[\mathrm{P}_{(\mathrm{PC})}\right] \cdot\left[\mathrm{D}_{(\mathrm{PyrPC})}\right]+k_{3} \cdot\left[\mathrm{P}_{(\mathrm{PI})}\right] \cdot\left[\mathrm{D}_{(\mathrm{Pyr} \mathrm{PC})}\right]}
$$

Since $\left[\mathrm{D}_{(\mathrm{Pyr} \mathrm{PC})}\right]$ equals $\left[\mathrm{D}_{(\mathrm{Pyr} \mathrm{PI})}\right]$

$\frac{V_{\mathrm{PyrPI}}}{V_{\mathrm{Pyy}}}=\frac{k_{2} \cdot\left[\mathrm{P}_{(\mathrm{PC})}\right]+k_{4} \cdot\left[\mathrm{P}_{(\mathrm{PI})}\right]}{k_{1} \cdot\left[\mathrm{P}_{(\mathrm{PC})}\right]+k_{3} \cdot\left[\mathrm{P}_{(\mathrm{PI})}\right]}$

When the acceptor vesicles consist of pure PI $\left(\left[\mathrm{P}_{(\mathrm{PC})}\right]=0\right)$ Eqn. A-6 is converted into Eqn. A-7

$\frac{V_{(\mathrm{Pyr} P \mathrm{P})}}{V_{(\mathrm{PyrPC})}}=\frac{k_{4}}{k_{3}}$

When the acceptor vesicles consist of pure PC $\left(\left[\mathrm{P}_{(\mathrm{PI})}\right]=0\right)$ Eqn. A-6 is converted into Eqn. A-8

$\frac{V_{(\mathrm{Pyr} P I)}}{V_{(\mathrm{Pyr} P C)}}=\frac{k_{2}}{k_{1}}$

When the acceptor vesicles consist of both $\mathrm{PC}$ and $\mathrm{PI}$, the $\left[\mathrm{P}_{\mathrm{PI}}\right] /\left[\mathrm{P}_{\mathrm{PC}}\right]$ ratio can be derived from Eqn. A-9

$\frac{\left[\mathrm{P}_{(\mathrm{PI})}\right]}{\left[\overline{\mathrm{P}}_{(\mathrm{PC})}\right]}=K_{\mathrm{rel}} \cdot \frac{\left[\mathrm{PI}_{\mathrm{Acc}}+\mathrm{PI}_{\mathrm{donor}}\right]}{\left[\mathrm{PC}_{\mathrm{Acc}}+\mathrm{PC}_{\mathrm{donor}}\right]}$

where the $K_{\text {rel }}$ represents the relative affinity of PI-transfer protein for yeast PI relative to egg PC (i.e. $K_{\text {rel }}=15.6$; see Table I). Since under our assay conditions the acceptor vesicles are present in large excess over the donor vesicles, the relative amounts of PI and PC bound to the transfer protein are determined by the lipid composition of the aceptor vesicles. Hence, Eqn. A-9 can be expressed as

$\frac{\left[\mathrm{P}_{(\mathrm{PI})}\right]}{\left[\mathrm{P}_{(\mathrm{PC})}\right]} \approx K_{\mathrm{rel}} \cdot \frac{\left[\mathrm{PI}_{\mathrm{Acc}}\right]}{\left[\mathrm{PC}_{\mathrm{Acc}}\right]}$

By measuring the relative transfer rates of PyrPI and PyrPC to acceptor vesicles of different PI-PC content, the rate constants of Eqns. A-1-A-4 can be estimated (see Table II, Results). 
By measuring the relative transfer rates of PyrPI and PyrPC to acceptor vesicles of different PI-PC content, the rate constants of Eqns. A-1-A-4 can be estimated (see Table II, Results).

\section{Acknowledgements}

This research was supported by grants from the Finnish Academy, the European Molecular Biology Organization (EMBO) and the Federation of Biological Sciences (FEBS) to P.J. Somerharju.

\section{References}

1 Wilgram, G.F. and Kennedy, E.R. (1963) J. Biol. Chem. 238, 2615-2619

2 Dawson, R.M.C. (1973) Subcell. Biochem. 2, 69-89

3 Morré, D.J., Kartenbeck, J. and Franke, W. (1979) Biochim. Biophys. Acta 559, 71-152

4 Morré, D.J. (1977) Cell. Surf. Rev. 4, 1-83

5 Wirtz, K.W.A. (1982) in Lipid-Protein Interactions (Jost, P.C. and Griffith, O.H., eds.), pp. 151-231, Wiley Interscience, New York

6 Kader, J.C., Douady, D. and Mazliak, P. (1982) in Phospholipids (Hawthorne, J.N. and Ansell, G.B., eds.), pp. 279-311, Elsevier Biomedical Press, Amsterdam

7 Kaplan, M.R. and Simoni, R.D. (1985) J. Cell Biol. 101, 441-445

8 Daum, G., Heidorn, E. and Paltauf, F. (1986) Biochim. Biophys. Acta 878, 93-101

9 Kaplan, M.R. and Simoni, R.D. (1985) J. Cell Biol. 101, 446-453

10 Molitoris, B.A. and Simon, F.R. (1986) J. Membrane Biol. $94,47-53$

11 Berridge, M.J. and Irvine, R.F. (1984) Nature 312, 315-321

12 Downes, C.P. and Michell, R.H. (1985) in Molecular Mechanisms of Transmembrane Signalling (Cohen, P. and Houslay, M.D., eds.), pp. 3-56, Elsevier, Amsterdam

13 Helmkamp, G.M., Jr., Harvey, M.S. Wirtz, K.W.A. and Van Deenen, L.L.M. (1974) J. Biol. Chem. 249, 6382-6389

14 DiCorleto, P.E., Warach, J.B. and Zilversmit, D.B. (1979) J. Biol. Chem. 254, 7795-7802

15 Daum, G. and Paltauf, F. (1984) Biochim. Biophys. Acta $784,385-391$
16 George, P.Y. and Helmkamp, G.M., Jr. (1985) Biochim. Biophys. Acta 836, 176-184

17 Demel, R.A., Kalsbeek, R., Wirtz, K.W.A. and Van Deenen, L.L.M. (1977) Biochim. Biophys. Acta 466, 10-22

18 Somerharju, P., Van Paridon, P.A. and Wirtz, K.W.A. (1983) Biochim. Biophys. Acta 731, 186-195

19 Helmkamp, G.M., Jr. (1985) Chem. Phys. Lipids 38, 3-16

20 Zborowski, J. and Demel, R.A. (1982) Biochim. Biophys. Acta 688, 381-387

21 Helmkamp, G.M., Jr., Wirtz, K.W.A. and Van Deenen, L.L.M. (1976) Arch. Biochem. Biophys. 174, 592-602

22 VanParidon, P.A., Visser, A.J.W.G. and Wirtz, K.W.A. (1987) Biochim. Biophys. Acta 898, 172-180

23 Kasper, A.M. and Helmkamp, G.M., Jr. (1981) Biochim. Biophys. Acta 664, 22-32

24 Trevelyan, W.G. (1966) J. Lipid Res. 7, 445-447

25 Somerharju, P. and Wirtz, K.W.A. (1982) Chem. Phys. Lipids 30, 81-91

26 Somerharju, P.J., Virtanen, J.A., Eklund, K.K., Vaino, P. and Kinnunen, P.K.J. (1985) Biochemistry 24, 2773-2781

27 Van Duijn, G., Dekker, J., Leunissen-Bijvelt, J., Verkley, A.J. and De Kruijff, B. (1985) Biochemistry 24, 7640-7650

28 Rouser, G., Fleischer, S. and Yamanioto, A. (1970) Lipids 5, 494-496

29 Batzri, S. and Korn, E.D. (1973) Biochim. Biophys. Acta 298, 2015-1019

30 Somerharju, P., Brockerhoff, H. and Wirtz, K.W.A. (1981) Biochim. Biophys. Acta 649, 521-528

31 Low, M.G. and Zilversmit, D.B. (1980) Biochim. Biophys. Acta 596, 223-234

32 Schermoly, M.J. and Helmkamp, G.M., Jr. (1983) Brain Res. 268, 197-200

33 Helmkamp, G.M., Jr., Nelemans, S.A. and Wirtz, K.W.A. (1976) Biochim. Biophys. Acta 424, 168-182

34 White, D.A. (1973) in Form and Function of Phospholipids 2nd Edn., (Ansell, G.B., Dawson, R.M.C. and Hawthorne, J.M., eds.), pp. 441-482, Elsevier, Amsterdam

35 Harrington, C.A. and Eichberg, J. (1983) J. Biol. Chem. 258, 2087-2090

36 Jelsema, C.L. and Morré, D.J. (1978) J. Biol. Chem. 253, $7960-7971$

37 Imai, A. and Gershengorn, M.C. (1987) Nature 325, $726-728$ 